\title{
Should the ApoE genotype be a covariate for clinical trials in Alzheimer disease?
}

\author{
Martin R Farlow*
}

\begin{abstract}
Should the apolipoprotein E (ApoE) genotype be a covariate for clinical trials in Alzheimer disease (AD)? ApoE is a transport protein for lipids, amyloid-beta proteins, and the different phenotypes differentially affect amyloid-beta deposition, neurofibrillary tangle formation, and microglial activation. The ApoE genotype has not affected efficacy in short symptomatic AD trials. ApoE4 has been associated with greater efficacy in at least two mild cognitive impairment studies. Vasogenic edema was more frequent in ApoE4 AD patients treated with a monoclonal antibody to amyloid beta. Since there is evidence that the ApoE genotype may differentially affect disease mechanisms, efficacy, and adverse effects in both AD and mild cognitive impairment trials, the ApoE genotype should be included as a covariate in future studies.
\end{abstract}

In clinical drug trials, it is appropriate to include as a covariate (a) any factor that is likely to differentially affect underlying rates of disease progression or (b) factors that are likely to differentially affect potential known mechanisms of action of the drug being studied or (c) both. With regard to therapeutic drug trials in Alzheimer disease $(\mathrm{AD})$, a frequent and appropriate concern is whether the apolipoprotein E (ApoE) genotype should be taken as a covariate in statistical analyses regarding efficacy or adverse effects. The ApoE $\varepsilon 4$ genotype is the major genetic risk factor identified for $\mathrm{AD}$. The ApoE $\varepsilon 4$ genotype is associated with earlier age of onset for the disease, with heterozygous patients having a $50 \%$ chance of dementia in their mid to late 70 s and homozygous patients having a $50 \%$ chance of dementia in their mid to late $60 \mathrm{~s}$. The odds ratio for developing dementia also goes

*Correspondence: mfarlow@iupui.edu

Department of Neurology, Indiana University School of Medicine, 541 Clinical Drive, CL 291, Indianapolis, IN 46202, USA up significantly with gene dose. These associations hold true for both sporadic and familial forms of the illness. Pathologically, inheritance of the E4 genotype has been associated with greater total amyloid deposition in the brain, both in cortical plaques and in the vessels, and increased neurofibrillary tangles $[1,2]$. In mild cognitive impairment $(\mathrm{MCI})$ or very-early-stage $\mathrm{AD}$, the $\mathrm{E} 4$ genotype is associated with greater deficits on the New York Paragraph Recall test, Auditory-Verbal Learning Test, and Buschke test at baseline. On magnetic resonance imaging, there are increases in both hippocampal atrophy and global atrophy at baseline [3], and on positron emission tomography using FDG (fluorine-18fluorodeoxyglucose), there are greater deficits in glucose metabolism in the posterior parietal and parahippocampal regions. In MCI subjects with the E4 genotype, cerebrospinal fluid analyses typically have shown decreased levels of the protein amyloid beta (A $\beta$ ) 1-42 and increased levels of Tau and pTau [4].

With regard to mechanisms of action with potential relevance to $\mathrm{AD}$, the ApoE protein has been demonstrated to have several functions, which include its roles as a major cholesterol-carrying protein in plasma and as the primary lipid-carrying transport protein in the central nervous system [5]. The ApoE protein also functions as a major transporter for the $A \beta$ proteins [6]. The ApoE2, E3, and E4 protein phenotypes have differential affinities for lipids and $A \beta$ and consequent variable efficiencies in these transport roles. There is evidence in neuropathological studies of $\mathrm{AD}$ patients of greater microglial activation around $A \beta$ plaques in patients with the ApoE 4 genotype [7]. These patients also demonstrate greater abnormal phosphorylation of Tau. In summary, the different ApoE2, E3, and E4 genotypes code for different ApoE protein phenotypes, which result in functional variation in the actions of this protein as it plays roles that virtually span known and theorized disease mechanisms involved in the pathogenesis or progression (or both) in AD.

With regard to the influence of the ApoE genotype on clinical factors of potential importance in AD clinical trials, there may be differential effects related to disease stage, trial length, and the specific effects of the drug 
regarding symptomatic actions versus effects on longerterm biological progression of the disease. In the original 12- to 26-week symptomatic trials of tacrine in mild to moderate AD, post hoc statistical analyses using the ApoE genotype to define subgroups of patients suggested that patients with the ApoE4 genotype progressed more rapidly on placebo and conversely were more likely to respond to treatment with tacrine [8]. However, later studies looking at the ApoE genotype as a factor in other cholinesterase inhibitor trials in mild to moderate AD (donepezil, rivastigmine, and galantamine) failed to confirm this hypothesized association [9-11].

Other short-term trials (6 weeks to 6 months) in mildto moderate-stage AD with drugs believed to beneficially affect mitochondrial function, energy metabolism, and/ or insulin resistance (caprylidene and rosiglitazone) were not positive overall, but subanalyses suggested that the subgroups with the ApoE2 or ApoE3 genotypes did significantly benefit [12]. However, with rosiglitazone, three later double-blind placebo-controlled studies of 1-year duration failed to reproduce or demonstrate beneficial effects in either the general AD population or the ApoE2 or ApoE3 genotype subgroups [13]. Overall, the influence of the ApoE genotype in shorter symptomatic studies in $\mathrm{AD}$ has been preliminary, negative, or equivocal.

Increased attention is being focused on an earlier stage of $\mathrm{AD}, \mathrm{MCI}$, in which several longer-duration trials have evaluated candidate drugs for delaying or preventing conversion from MCI to AD. The ApoE genotype does appear to play a more significant role in at least some of the longer MCI trials. In the Alzheimer Disease Cooperative Study group's MCI double-blind placebocontrolled trial (duration of 3 to 4 years) of donepezil or vitamin E versus placebo, MCI patients with the ApoE4 genotype on placebo were much more likely to progress and convert to AD [14]. Patients with the ApoE4 genotype showed significantly lower rates of conversion from MCI to AD versus placebo when treated with donepezil. Similar findings were seen in a large comparably designed double-blind placebo-controlled trial of rivastigmine in MCI. Thus, substantial evidence suggests that ApoE4related differences in brain and disease progression may be observed in association with ApoE4 in MCI and that these effects may differentially influence detectable treatment effects.

The ApoE genotype also appears to have had differential influence on adverse effects in a phase II AD treatment trial of a monoclonal antibody against $A \beta$ (bapineuzumab). In this trial, subjects with the ApoE4 genotype were much more likely to develop vasogenic brain edema at lower doses of this antibody [15]. The known greater $A \beta$ deposition in cerebral vasculature in $\mathrm{AD}$ patients with the ApoE4 genotype may or may not play a role in this phenomenon.
In summary, there is evidence that, through a variety of interactions and functional effects, variation in the ApoE genotype and the coded-for, different resultant polymorphic proteins may affect rates of disease progression in $\mathrm{AD}$ and potentially responses to therapies (with regard to both efficacy and safety). It therefore would seem prudent to stratify or include the ApoE genotype as a covariate in most AD therapeutic trials. Inclusion of the ApoE genotype should decrease variance in future studies, increasing the likelihood of successfully determining whether the drug under study benefits patients or is safe in treating $\mathrm{AD}$.

\section{Abbreviations}

$A \beta$, amyloid beta; AD, Alzheimer disease; ApoE, apolipoprotein E; MCl, mild cognitive impairment.

\section{Competing interests}

The author receives research funding from Bristol-Myers Squibb Company (Princeton, NJ, USA), Danone (Paris, France), Elan Corporation (Dublin, Ireland), Eli Lilly and Company (Indianapolis, IN, USA), Forest Pharmaceuticals, Inc. (St. Louis, MO, USA), Pfizer Inc (New York, NY, USA), Novartis International AG (Basel, Switzerland), Octapharma AG (Lachen, Switzerland), and Sonexa Therapeutics, Inc. (San Diego, CA, USA); receives honoraria as a scientific consultant for Adamas Pharmaceuticals, Inc. (Emeryville, CA, USA), Accera, Inc. (Broomfield, CO, USA), Adlyfe Inc. (Rockville, MD, USA), AstraZeneca (London, UK), Astellas Pharma Inc. (Tokyo, Japan), Bayer Corp. (Emeryville, CA, USA), BioRx (Cincinnati, OH, USA), CoMentis Inc. (South San Francisco, CA, USA), Cortex Pharmaceuticals, Inc. (Irvine, CA, USA), Eisai Inc. (Woodcliff Lake, NJ, USA), Dainippon Sumitomo Pharma Co., Ltd. (Osaka, Japan), El Lilly and Company, GE Healthcare Inc. (Princeton, NJ, USA), GlaxoSmithKline (Uxbridge, Middlesex, UK), Medivation, Inc. (San Francisco, CA, USA), Merck (Darmstadt, Germany), Novartis International AG, Noven, Octapharma AG, Prana Biotechnology Ltd (Melbourne, Australia), QR Pharma, Inc. (Radnor, PA, USA), sanofi-aventis (Paris, France), Schering-Plough Corporation (Kenilworth, NJ, USA), Suven Life Sciences Ltd. (Hyderabad, India), and Toyama Chemical Co. Ltd (Tokyo, Japan); and is a speaker for Eisai Inc., Forest Pharmaceuticals, Inc., Janssen (Titusville, NJ, USA), Pfizer Inc, and Novartis International AG.

\section{Acknowledgments}

This work was supported by grant P30 AG10133 of the US Public Health Service National Institutes of Health/National Institute on Aging.

Published: 8 June 2010

\section{References}

1. Premkumar DR, Cohen DL, Hedera P, Friedland RP, Kalaria RN: Apolipoprotein E-episilon4 alleles in cerebral amyloid angiopathy and cerebrovascular pathology associated with Alzheimer's disease. Am J Pathol 1996, 148:2083-2095.

2. Ghebremedhin E, Schultz C, Braak E, Braak H: High frequency of apolipoprotein $\mathrm{E}$ epsilo4 allele in young individuals with very mild Alzheimer's disease-related neurofibrillary changes. Eur Neurol 1998 153910:152-155

3. Farlow MR, He Y, Tekin $\mathrm{S}, \mathrm{Xu}$ J, Lane R, Charles HC: Impact of APOE in mild cognitive impairment. Neurol 2004, 63:1898-1901.

4. Buerger K, Teipel SJ, Zinkowski R, Sunderland T, Andreasen N, Blennow K, Ewers M, DeBernardis J, Shen Y, Kerkman, Du Y, Hampel H: Increased levels of CSF phosphorylated tau in apolipoprotein E epsilon 4 carriers with mild cognitive impairment. Neuroscience Letters 2005, 391:48-50.

5. Linton MF, Gish R, Hubl ST, Butler E, Esquivel C, Bry WI, Boyles JK, Wardell MR, Young SG: Phenotypes of apolipoprotein B and apolipoprotein E after liver transplantation. J Clin Invest 1991, 88:207-281.

6. Tokuda T, Calero M, Matsubara E, Vidal R, Kumar A, Permanne B, Zlokovic B, Smith JD, Ladu MJ, Rostagno A, Frangione B, Ghiso J: Lipidation of apolipoprotein $E$ influences its isoform-specific interaction with Alzheimer's amyloid beta peptides. Biochem J 2000, 348:359-365. 
7. Egensperger R, Kosel S, von Eitzen U, Graeber MB: Microglial activation in Alzheimer disease: association with APOE genotype. Brain Pathol 2006, 8:439-447.

8. Poirier J, Delisle M-C, Quirion R, Aubert I, Farlow M, Lahiri D, Hui S, Bertrand P, Nalbantoglu J, Gilfix BM, Gauthier S: Apolipoprotein E4 allele as a predictor of cholinergic deficits and treatment outcome in Alzheimer's disease. Proc Natl Acad SciU S A 1995, 92:12260-12264.

9. Rigaud A-S, Traykov L, Latour F, Couderc R, Moulin F, Forette F: Presence or absence of at least one e4 allele and gender are not predictive for the response to donepezil treatment in Alzheimer's disease. Pharmacogenom 2002, 12:415-420

10. Blesa R, Aguilar M, Casanova JP, Boada M, Martinez S, Alom J, Hernandez de la Hoz C, Sancho J, Fernandez O, Gil-Neciga E, Masso JFM: Relationship between the efficacy of rivastigmine and apolipoprotein $E(\varepsilon 4)$ in patients with mild to moderately severe Alzheimer disease. Alz Dis Assoc Dis 2006, 20:248-254.

11. Aerssens J, Raeymaekers P, Lilienfeld S, Geerts H, Konings F, Parys W: APOE genotype: no influence on galantamine treatment efficacy or on rate of decline in Alzheimer's disease. Dement Geriatr Cogn Disord 2001, 12:69-77.

12. Roses AD: The medical and economic roles of pipeline pharmacogenetics: Alzheimer's disease as a model of efficacy and HLA-B(*)5791 as a model of safety. Neuropsychopharmacology 2009, 34:6-17

13. Alzforum: Drugs in Clinical Trials - Rosiglitazone [http://www.alzforum.org/ $\mathrm{drg} / \mathrm{drc} /$ detail.asp?id=116].

14. Petersen RC, Thomas RG, Grundman M, Bennett D, Doody R, Ferris S, Galasko D, Jin S, Kaye J, Levey A, Pfeiffer E, Sano M, van Dyck CH, Thal LJ, for the Alzheimer's Disease Cooperative Study Group: Vitamin E and donepezil for the treatment of mild cognitive impairment. N Eng/ J Med 2005, 352:2379-2388.

15. Salloway S, Sperling R, Gilman S, Fox NC, Blennow K, Raskind M, Sabbagh M, Honig LS, Doody R, van Dyck CH, Mulnard R, Barakos J, Gregg KM, Liu E, Lieberburg I, Schenk D, Black R, Grundman M; Bapineuzumab 201 Clinical Trial Investigators: A phase 2 multiple ascending dose trial of Bapineuzumab in mild to moderate Alzheimer disease. Neurol 2009, 73:2061-2070

doi:10.1186/alzrt39

Cite this article as: Farlow MR: Should the ApoE genotype be a covariate for clinical trials in Alzheimer disease? Alzheimer's Research \& Therapy 2010, 2:15. 